\title{
ASTEROID SIZING BY RADIOGALAXY OCCULTATION AT 5 GHZ
}

\author{
Lehtinen, $\mathrm{K}$.
}

2016-05-10

Lehtinen , K , Bach , U , Muinonen , K, Poutanen , M \& Petrov , L 2016 , ' ASTEROID

SIZING BY RADIOGALAXY OCCULTATION AT $5 \mathrm{GHZ}$ ' , Astrophysical Journal Letters , vol. 822 , no. 2 , 21 . https://doi.org/10.3847/2041-8205/822/2/L21

http://hdl.handle.net/10138/183627

https://doi.org/10.3847/2041-8205/822/2/L21

unspecified

publishedVersion

Downloaded from Helda, University of Helsinki institutional repository.

This is an electronic reprint of the original article.

This reprint may differ from the original in pagination and typographic detail.

Please cite the original version. 


\title{
ASTEROID SIZING BY RADIOGALAXY OCCULTATION AT 5 GHZ
}

\author{
K. Lehtinen ${ }^{1}$, U. Bach ${ }^{2}$, K. Muinonen ${ }^{1,3}$, M. Poutanen ${ }^{1}$, and L. Petrov ${ }^{4}$ \\ ${ }^{1}$ Finnish Geospatial Research Institute FGI, Geodeetinrinne 2, FI-02430 Masala, Finland; kimmo.lehtinen@nls.fi \\ ${ }^{2}$ Max-Planck-Institut für Radioastronomie, Radioobservatorium Effelsberg, Max-Planck-Str. 28, D-53902 Bad Münstereifel-Effelsberg, Germany \\ ${ }^{3}$ Department of Physics, P.O. Box 64, University of Helsinki, FI-00014 Helsinki, Finland \\ ${ }_{4}^{4}$ Astrogeo Center, Falls Church, VA 22043, USA \\ Received 2016 February 15; revised 2016 April 15; accepted 2016 April 17; published 2016 May 2
}

\begin{abstract}
Stellar occultations by asteroids observed at visual wavelengths have been an important tool for studying the size and shape of asteroids and for revising the orbital parameters of asteroids. At radio frequencies, a shadow of an asteroid on the Earth is dominated by diffraction effects. Here, we show, for the first time, that a single observation of an occultation of a compact radio source at a frequency of $5 \mathrm{GHz}$ can be used to derive the effective size of the occulting object and to derive the distance between the observer and the center of the occultation path on the Earth. The derived diameter of the occulting object, asteroid (115) Thyra, is $75 \pm 6 \mathrm{~km}$. The observed occultation profile shows features that cannot be explained by diffraction of a single asteroid.
\end{abstract}

Key words: minor planets, asteroids: individual (Thyra) - occultations

\section{INTRODUCTION}

Occultation is a phenomenon where a distant object, usually a star, is hidden by the Moon, a planet, or its ring or a small body in our solar system. At visual wavelengths, a shadow of a main-belt asteroid on the Earth during an occultation closely resembles its true silhouette on the plane of the sky. Thus, the shape and size of an asteroid can be deduced if there are several simultaneous observations of an occultation made at different distances from the center of an occultation path. A single observation of an occultation at visual wavelengths gives only a lower limit for the size of an asteroid. In this Letter, we show that it is possible to deduce the effective size of an asteroid from a single observation of an occultation at radio wavelengths by modeling the occultation with FresnelKirchhoff diffraction.

During an occultation, the limb of an asteroid works as a sharp edge for the plane wave coming from the occulted object, producing diffraction that is mathematically expressed by the Fresnel-Kirchhoff diffraction formula for monochromatic radiation. The visibility of diffraction fringes on the shadow of the occulter is governed by the value of the Fresnel number $F=r^{2} /(D \lambda)$, where $r$ is the radius of the occulter, $D$ is the distance between the occulter and the observer, and $\lambda$ is the wavelength of radiation. The case $F \gtrsim 1$ corresponds to the regime of Fresnel diffraction, where the shadow of the occulter on the Earth closely resembles its silhouette because on a typical observation, the diffraction effects are negligible compared to the noise of an observation. At visual wavelengths, occultations by main-belt asteroids are in the region of Fresnel diffraction. The case $F \lesssim 1$ corresponds to the regime of Fraunhofer diffraction, where the diffraction pattern of an occultation is larger than the physical size of the occulter and dominated by interference fringes. This condition is fulfilled when observing an occultation at radio wavelengths. In the case of our observation of the asteroid (115) Thyra, $F \approx 0.06$. The characteristic scale of a diffraction pattern is given by the Fresnel scale $F_{s}=\sqrt{\lambda D / 2}$. Also, other definitions for $F_{s}$ have been used, such as $\sqrt{(\lambda D) /(2 \pi)}$ and $\sqrt{\lambda D}$. In the Fraunhofer region, if the diameter of the occulter is less than about the Fresnel scale, the Fresnel scale determines the size of a diffraction pattern on the Earth, not the physical size of the occulter. In this case, the distance between the first interference fringe maxima of the diffraction pattern (the size of the Airy ring) is $2 \sqrt{3}$ in Fresnel scale units. In practice, this enables one to get the distance of the occulter if the size of the shadow on the Earth is known. The size of the occulter mainly affects the amplitude of the interference fringes of the diffraction pattern.

In principle, if the diffraction effects are significant, one can derive both the size of the occulter and its distance without an independent measurement of the size (that is, the velocity) of the shadow (Cooray 2003). In practice, this requires that both the projected size of the occulted object on the sky and the occultation light curve are known with a better than $~ 5 \%$ accuracy.

\section{OCCULTATION PREDICTIONS}

The occultation predictions were made with the LinOccult ${ }^{5}$ program, a free program made by A. Plekhanov. The use of LinOccult requires three auxiliary data sets. First, for the positions of the background objects, we use the latest Radio Fundamental Catalog, available at http://astrogeo.org/rfc, hereafter Astrogeo (L. Petrov \& Y. Y. Kovalev 2016, in preparation; Petrov et al. 2008 and references therein). Second, we obtain the asteroid orbital elements from the latest astorb.dat ${ }^{6}$ file. Third, we use the JPL ephemerides ${ }^{7}$ for the major planets.

Thyra was predicted to occult the radio source J0014+0854 on 2015 March 23 at 09:43:32 UT. The distance of the Effelsberg radio telescope from the center of the occultation path was predicted to be $70 \pm 90 \mathrm{~km}$. The velocity of the shadow of Thyra on the Earth was $52.5 \mathrm{~km} \mathrm{~s}^{-1}$ during the occultation.

\section{OBSERVATIONS}

The receiver used for the observations at the Effelsberg radio telescope was chosen to be the "S60mm Double Beam"

\footnotetext{
http://andyplekhanov.narod.ru/occult/occult.htm

$6 \mathrm{ftp} / / / \mathrm{ftp}$. lowell.edu/pub/elgb

7 The file $\ln x 1600 \mathrm{p} 2200.405$ is available at ftp://ssd.jpl.nasa.gov/pub/ eph/planets/Linux/de405.
} 
receiver: first, the frequencies at $\lesssim 20 \mathrm{GHz}$ are less affected by variable atmospheric emission and absorption than at higher frequencies; second, it has the lowest noise of the receivers in the $\lesssim 20 \mathrm{GHz}$ frequency range, having rms noise of about $1.4 \mathrm{mJy}$ in $1 \mathrm{~s}$ of integration time and with $500 \mathrm{MHz}$ bandwidth.

The frequency range of the receiver is $\sim 4.6-5.1 \mathrm{GHz}$. We use the measured bandpass of the receiver to derive an effective frequency of $4.79 \mathrm{GHz}$. A signal from a noise diode calibrator, with a noise temperature of $1.8 \mathrm{~K}$, is periodically inserted into the measured signal, giving the relation between temperature and instrumental units. The antenna temperatures were converted to Jansky units using an observation of the flux density calibrator $3 \mathrm{C} 48$. The derived sensitivity of $1.56 \mathrm{~K} \mathrm{Jy}^{-1}$ is in good agreement with the theoretical estimate of $1.55 \mathrm{~K} \mathrm{Jy}^{-1}$. The flux density was corrected for atmospheric absorption using an estimated opacity of 0.018 at $4.79 \mathrm{GHz}$, which is a typical value at Effelsberg. The original data were averaged over two samples to give a time resolution of about $0.03 \mathrm{~s}$. The standard deviation of the occultation profile is $\approx 8 \mathrm{mK}$, corresponding to $\approx 5 \mathrm{mJy}$.

\section{ASTEROID (115) THYRA}

Thyra is a main-belt asteroid, categorized as a stony S-type asteroid (DeMeo et al. 2009). The effective diameter of Thyra is estimated to be $79.8 \pm 1.4 \mathrm{~km}$ (Tedesco et al. 2004), derived by modeling IRAS infrared data with a refined Standard Thermal Model (STM; Lebofsky et al. 1986). Based on thermophysical modeling (Delbò et al. 2015 and references therein) of IRAS data using shape and spin vector solution of Thyra from light curve inversion (Michalowski et al. 2004), the equivalent diameter of Thyra is estimated to be $92 \pm 2 \mathrm{~km}$ (Delbò \& Tanga 2009). The AKARI mid-IR asteroid survey gives a mean diameter of $80.7 \pm 0.9 \mathrm{~km}$ for Thyra (Usui et al. 2011), based on STM. The geocentric distance and angular size (assuming a diameter of $80 \mathrm{~km}$ ) of Thyra were $\sim 2.97$ au and 0 ". 037 during the occultation.

The Database of Asteroid Models from Inversion Techniques $(\mathrm{DAMIT})^{8}$ gives a three-dimensional model of Thyra from light curve inversion (Durech et al. 2010). We use the web interface of the DAMIT to obtain a model sky projection of Thyra at the time of the occultation, shown in Figure 1. This projection is used to make the silhouette of Thyra, which is used in Fraunhofer diffraction model to calculate the diffraction pattern (see Section 6).

\section{RADIO SOURCE J0014+0854}

The coordinates of J0014+0854 are R.A. $(\mathrm{J} 2000)=00: 14: 19.7400, \operatorname{decl} .(\mathrm{J} 2000)=+08: 54: 02.0484$, with a semimajor axis of the error ellipse of 0.59 mas (Astrogeo). J0014+0854 was observed on 2014 February 3 and 2015 October 19 and 30 with the Very Long Baseline Array (VLBA) at $4.3 \mathrm{GHz}$ (C-band) and $7.6 \mathrm{GHz}$ (X-band) in the framework of the wide-field calibrator survey VCS8/9 (L. Petrov 2016, in preparation). The total integration time was $60 \mathrm{~s}$ in 2014 and $180 \mathrm{~s}$ in 2015. The two last epochs were combined for imaging. The integral flux density at $4.3 \mathrm{GHz}$ was $43 \mathrm{mJy}$ in 2014 and $38 \mathrm{mJy}$ in 2015 . The source shows a typical core-jet morphology. The best Gaussian model of the

\footnotetext{
http://astro.troja.mff.cuni.cz/projects/damit
}

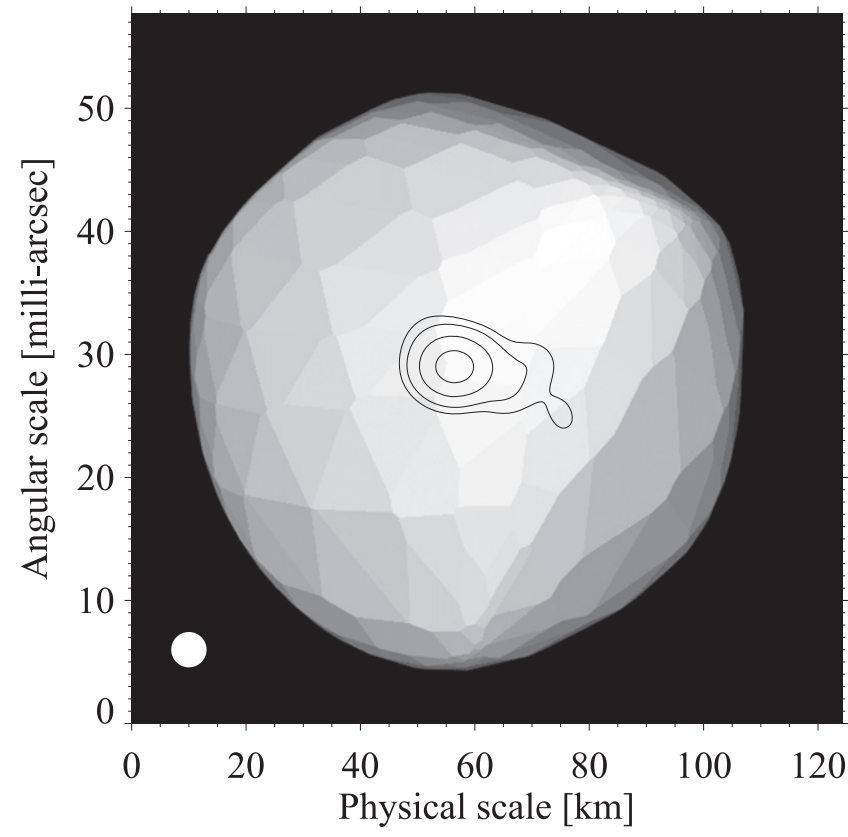

Figure 1. A 3D model of Thyra as seen by an observer on the Earth at the time of the occultation. The model, obtained from the DAMIT database (http:/ astro.troja.mff.cuni.cz/projects/damit), is based on light curve inversion. The contours show the structure of $\mathrm{J} 0014+0854$ as based on C-band $(4.3 \mathrm{GHz})$ VLBI observations on 2015 October (Astrogeo), with contour levels of $0.3,1$, 5 , and $20 \mathrm{mJy} /$ beam. The relative positions and orientations of J0014+0854 and Thyra do not follow their actual values at the time of the occultation. The FWHM size of the circular Gaussian restoring beam of the VLBI observations is shown in the lower left corner. Both the physical and angular scales are valid for Thyra, while for J0014+0854 and the beam only the angular scale is valid.

core has FWHM axes of $0.7 \times 0.8$ mas and peak flux density of $35 \mathrm{mJy} /$ beam. The smallest baseline length of the VLBA experiments was $2.4 \mathrm{M} \lambda$. Therefore, the C-band image accounts for all emission that comes from regions with characteristic sizes less than 80 mas. The integral flux density at $7.6 \mathrm{GHz}$ was $37 \mathrm{mJy}$ in 2014 an $33 \mathrm{mJy}$ in 2015 . We interpolate linearly the flux densities both in time and frequency to derive a flux density of $39 \mathrm{mJy}$ at $4.79 \mathrm{GHz}$ at the time of the occultation. A C-band image of J0014+0854 is shown in Figure 1. J0014+0854 is positionally coincident with an X-ray source MS0011.7+0837, which Gioia \& Luppino (1994) classify as a radiogalaxy. According to Rector et al. (1999), the source MS0011.7+0837, that is, J0014+0854, is a radiogalaxy or possibly a low-luminosity BL Lac object.

The FWHM beam size of the Effelsberg $100 \mathrm{~m}$ telescope at $4.79 \mathrm{GHz}$ is about $140^{\prime \prime}$. The VLA FIRST ${ }^{9}$ (Faint Images of the Radio Sky at Twenty-cm) survey at $\approx 1.4 \mathrm{GHz}$ reveals that, within a distance of $200^{\prime \prime}$ from the position of J0014+0854, there is one companion at a distance of 18 ". 7 from J0014+0854 with a flux density $25 \%$ of the main component that is probably a radiolobe. The flux density measured with the Effelsberg radio telescope is thus the total flux density from (at least) two sources. Thus, when deriving the relative change of intensity of J0014+0854 during the occultation, we use the flux density from VLBI observations, not the flux density measured with the Effelsberg telescope.

$\mathrm{J} 0014+0854$ was found to be linearly polarized by $6.6 \% \pm$ $0.4 \%$, with no change of polarization during the occultation.

9 http://sundog.stsci.edu 
We do not expect polarization to play an important role for the diffraction pattern (Muinonen 1989).

\section{RESULTS}

\subsection{Diffraction Models}

We perform model calculations of diffraction using three levels of complexity.

i. Spherical asteroid and a point-like occulted object. In this case, there is an analytic solution to the FresnelKirchhoff diffraction formula by using Lommel functions (Born \& Wolf 1980; Roques et al. 1987). If $r$ is the radius of the occulter, $S$ is the apparent separation between the occulter and the occulted object, both expressed in Fresnel scale units, the normalized flux density is

$$
I(r, S)=\left\{\begin{array}{l}
1+U_{1}^{2}(r, S)+U_{2}^{2}(r, S) \\
-2 U_{1}(r, S) \sin \left(\frac{1}{2} \pi\left(r^{2}+S^{2}\right)\right) \\
+2 U_{2}(r, S) \cos \left(\frac{1}{2} \pi\left(r^{2}+S^{2}\right)\right), S \geqslant r, \\
U_{0}^{2}(r, S)+U_{1}^{2}(r, S), S \leqslant r
\end{array}\right.
$$

where $U_{n}(r, S)$ are Lommel functions defined by

$$
U_{n}(r, S)=\sum_{k=0}^{\infty}(-1)^{k}(r / S)^{n+2 k} J_{n+2 k}(\pi r S)
$$

where $J_{n}$ is the bessel function of order $n$.

ii. Spherical asteroid and a spherical occulted object with a finite diameter. In this case, one convolves the diffraction pattern obtained above with the shape of the object. The effect is to reduce the amplitude of the interference fringes.

iii. An asteroid with an arbitrary shape and a point-like occulted object. In this case, we follow the formulation given by Trahan \& Hyland (2014), which is partly based on the work of Roques et al. (1987; see also Muinonen 1989). The sky projection of Thyra at the time of occultation (see Figure 1) is used to make a silhouette image of Thyra with a size of $256 \times 256$ pixels. The obtained shadow is shown in Figure 2. In view of the relatively low signal-to-noise ratio of our observations, we find that neither the true, finite size of the occulted object, nor the true shape of Thyra has a noticeable effect on the diffraction pattern. Thus, it is justified to make Fresnel-Kirchhoff diffraction calculations by assuming a point-like occulted object and a spherical asteroid. In general, the finite size of an occulted object has to be taken into account only when the apparent size of the object is larger than about the apparent size of the occulter.

The models above are valid in the case of monochromatic radiation. The difference between the diffraction pattern at the effective frequency and the pattern derived by integrating over the measured bandpass of the receiver was found to be $\sim 2 \%$ at maximum. Thus, all the model calculations have been made using monochromatic radiation.

\subsection{Modeling the Observation}

The observed occultation profile is shown in Figure 3; panel (a) shows the whole profile, while panel (b) shows the mean

\section{Relative intensity}
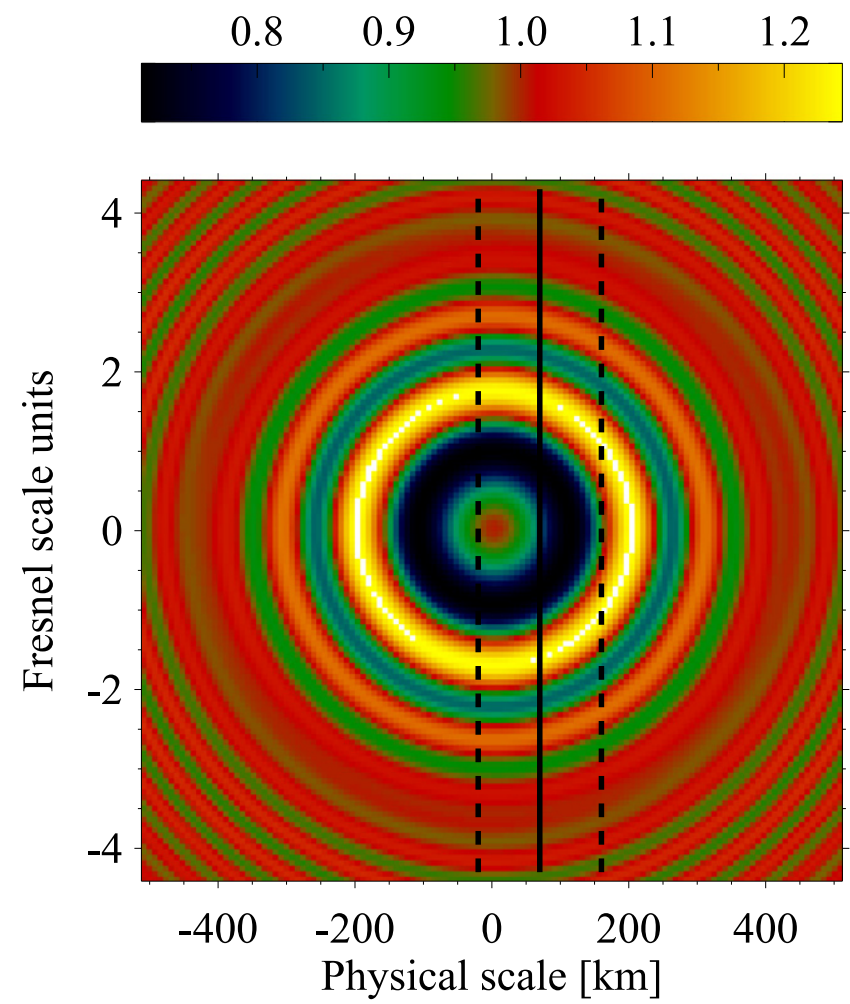

Figure 2. Theoretical diffraction pattern (shadow) of Thyra on the surface of Earth at the time of the occultation. The model is based on the actual silhouette of Thyra at the time of the occultation (Figure 1). Thus, small deviation from spherical symmetry can be seen. Color indicates the amount of relative change of flux density of the occulted object. The vertical black solid line is located at a distance of $70 \mathrm{~km}$ from the center of the occultation, corresponding to the predicted distance of Effelsberg radio telescope. The dashed lines indicate the uncertainty of the predicted distance.

value of the two halves located symmetrically around the center of the profile, giving a better signal-to-noise ratio. The main features of the observed profile are the following.

i. At the center of the occultation, there is a plateau where the relative intensity is close to unity.

ii. The first diffraction maxima are asymmetrical. On the edges facing the center of the occultation, the intensity changes more gradually than on the outer edges where the change of intensity is abrupt.

iii. The observed values of the first interference fringe peaks are about 1.4 in units of relative intensity. The double structure of the second positive interference fringe in Figure 3(b) may be caused by noise and we do not consider it further.

The free parameters in the occultation modeling are the size of the occulter, the distance of the observer from the center of the occultation path, and the time of the occultation. The size of the occulter and the distance of the observer from the center of the occultation path can be separated in the modeling because the former affects mainly the depth of the first interference fringe minima, while the latter affects mainly the level of the intensity at the center of the occultation and the size of the occultation profile. The model fitting was performed by using Levenberg-Marquardt least-squares fitting (Moré 1978; Markwardt 2009). The best model fit is shown superimposed in 

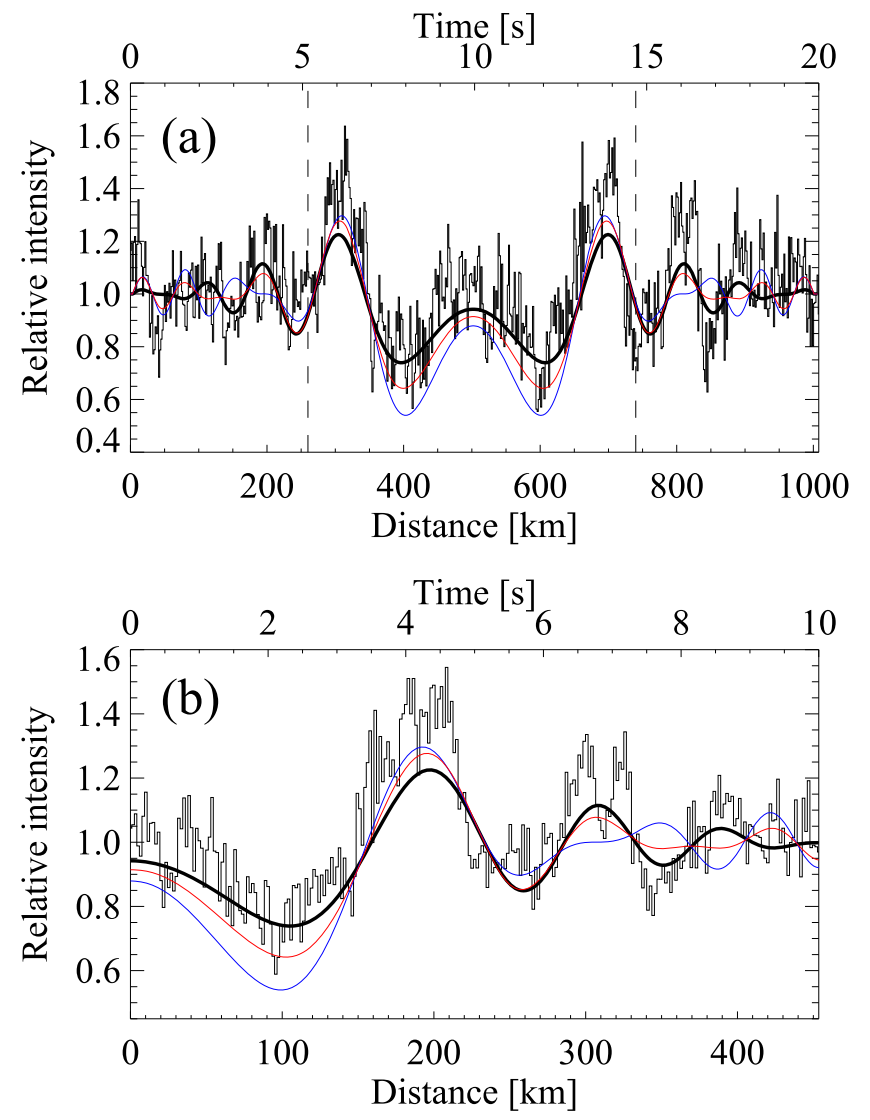

Figure 3. Observed occultation profile, showing the relative intensity of the occulted radiogalaxy as a function of time. Panel (a) shows the whole observed profile. The dashed lines delineate the region that has been used for model fitting. Time is in seconds after 09:43:17.25 UT. Panel (b) shows the profile after folding the profile in panel (a) through the center of the occultation. Distance is obtained by multiplying the time with the speed of the shadow on the Earth. The best-fit model of Fraunhofer diffraction, giving a diameter of $75 \mathrm{~km}$, is plotted with a thick solid line. The blue line shows the profile for a diameter of $110 \mathrm{~km}$, which gives the theoretical maximum of the first interference peaks (see Section 7). The red line shows the profile for a diameter of $92 \mathrm{~km}$, derived by thermophysical modeling (Delbò \& Tanga 2009; see Section 3).

Figure 3. The likelihoods of the diameter and the distance from the center of the occultation, after fixing the time, is shown in Figure 4, based on Bayesian estimation of parameters. The derived diameter of Thyra is $75 \pm 2 \mathrm{~km}$, the distance from the center of the occultation is $41 \pm 5 \mathrm{~km}$, and the time of the occultation is UT09:43:26.74 $\pm 0.03 \mathrm{~s}$. The uncertainties given are formal uncertainties of a single fit. We derive the total uncertainties of the model parameters by assuming a $10 \%$ uncertainty for VLBA observations, 5\% uncertainty for Effelsberg observations, and $10 \%$ uncertainty stemming from possible flux density variations of $\mathrm{J} 0014+0854$, totaling an uncertainty of $15 \%$ for the relative flux density of J0014+0854 during the occultation. Then, the derived diameter of Thyra varies from 69 to $81 \mathrm{~km}$. This is compatible with the diameter of about $80 \mathrm{~km}$, based on modeling IRAS or AKARI data with an STM (Tedesco et al. 2004; Usui et al. 2011). However, our result disagrees with the diameter of about $92 \mathrm{~km}$ (shown in Figure 3 as a red line), based on modeling IRAS data with a thermophysical model (Delbò \& Tanga 2009). The reduced chi-squared value of the fit is $\sim 81$. If fixing the diameter to $80 \mathrm{~km}$ or $92 \mathrm{~km}$, the chi-squared value is $\sim 86$ and $\sim 95$, respectively.

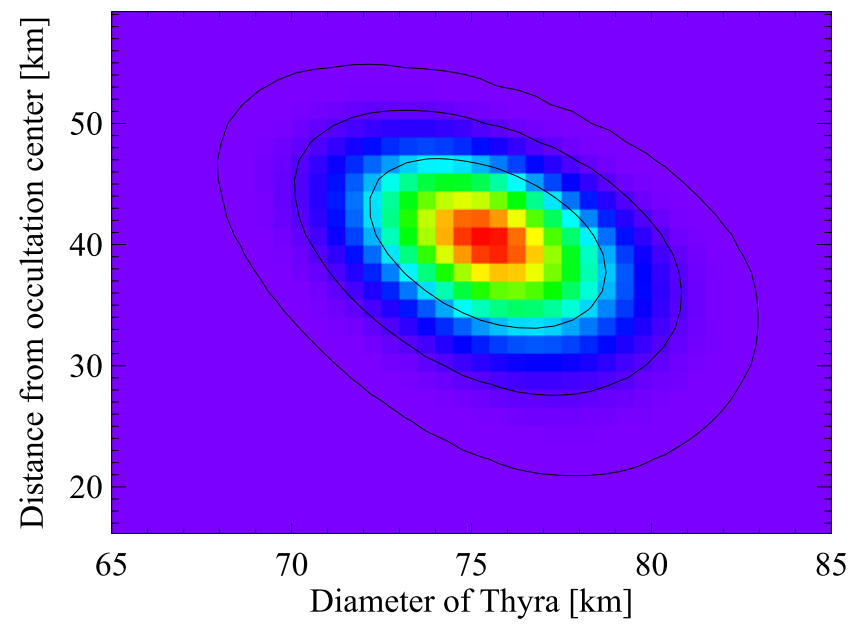

Figure 4. Likelihoods for two model parameters, the diameter of Thyra, and the distance between the observer and the center of the occultation path, after fixing the third model parameter, the time of the occultation. Contours show the $1 \sigma, 2 \sigma$, and $3 \sigma$ probability regions.

\section{DISCUSSION}

In general, the obtained fit is good, but it fails to fit in detail the maximum values of the interference fringes and the asymmetry of the first positive interference fringes. The maximum values of the interference fringes depend on the size of an occulter. We find that for a case of a spherical occulter and a point-like occulted object the theoretical maximum of the first interference peak, for the case of Thyra, is about 1.3, which is realized when the diameter of an occulter is similar to the Fresnel scale. For Thyra, that would correspond to a size of about $110 \mathrm{~km}$ (shown in Figure 3 as a blue line). However, such a large size is excluded on the basis of thermal and thermophysical modeling (Tedesco et al. 2004; Delbò \& Tanga 2009; Usui et al. 2011).

If the true flux density of J0014+0854 at the time of the occultation was greater than the estimated value, the maxima of interference peaks would be lower. Accordingly, the depth of the interference minima would be smaller, and the diameter of Thyra would be smaller than our current estimate. On the other hand, the derived diameter, $69-81 \mathrm{~km}$, is already on the lower side of the range of values derived by thermal and thermophysical modeling $(80-92 \mathrm{~km})$. Thus, we believe that the unexpectedly high intensities of the positive interference peaks are real and cannot be explained by possible flux density variations of $\mathrm{J} 0014+0854$ or calibration uncertainties.

Because our observation is in the Fraunhofer region, the discrepancy between theory and observation cannot be explained by any physically feasible shape of a single occulter. We propose two models that have a structure clearly different from a single occulter. The first model is a binary asteroid, consisting of two objects with similar sizes. The second, more speculative model consists of an asteroid surrounded by material, either in the form of a cloud or a ring. According to our calculations a small moon orbiting Thyra cannot explain the observed occultation profile. A detailed analysis of these models is beyond the scope of this Letter.

\section{CONCLUSIONS}

Modeling of occultations at radio wavelengths relies purely on the theory of Fresnel-Kirchhoff diffraction; thus, there is no 
need to have knowledge about an asteroid's physical properties, as is required in the case of thermal and thermophysical modeling. Taking our case as a typical example, the occultation method enables one to determine asteroid diameters with about $2 \mathrm{~km}$ uncertainty, provided that the flux density of the occulted object at the time of the occultation is accurately known. This is comparable to the nominal uncertainty achieved with thermal and thermophysical modeling of infrared data.

Compact radio sources are particularly well suited for occultation observations because their structure is known from VLBI observations, and thus the structure of the occulted object is not a free parameter in modeling of occultations.

Inspired by successful observations of Thyra, we consider two promising applications of such observations. First, we propose obtaining accurate positions of asteroids relative to radio sources and investigating the feasibility of using these measurements for aligning the dynamical reference system of solar system objects with the kinematic reference system defined by VLBI observations of extragalactic objects. Second, we are going to investigate the feasibility of a serendipitous search for Kuiper belt objects, as has been done at optical wavelengths (e.g., Bickerton et al. 2009; Schlichting et al. 2012; Liu et al. 2015). Radio observations have several advantages over optical occultations: (i) the shadow on Earth is larger; (ii) radiotelescopes are already equipped with standard recording systems that provide continuous and very fast data sampling; (iii) the flux density variations can be measured with an uncertainty up to $\approx 0.5 \%$ when a strong $1 \mathrm{Jy}$ radio source is observed with a setup similar to ours, a level that is hard to obtain in visual observations.

K.L. gratefully acknowledges funding from the Jenny and Antti Wihuri Foundation (www.wihurinrahasto.fi). K.M. is supported, in part, by the Academy of Finland (grant No. 257966). Based on observations with the $100 \mathrm{~m}$ telescope of the MPIfR (Max-Planck-Institut für Radioastronomie) at Effelsberg. We thank the $100 \mathrm{~m}$ Effelsberg scheduler, Dr. A. Kraus, for allocating the observing time for this project. We wish to thank Andrey Plekhanov for the freely available LinOccult program.

Facility: Effelsberg (S60mm Double Beam).

\section{REFERENCES}

Bickerton, S. J., Welch, D. L., \& Kavelaars, J. J. 2009, AJ, 137, 4270

Born, M., \& Wolf, E. 1980, Principles of Optics (6th ed.; New York: Pergamon)

Cooray, A. 2003, ApJL, 589, L97

Delbò, M., Müller, M., Emery, J. P., Rozitis, B., \& Capria, M. T. 2015, arXiv: 1508.05575

Delbò, M., \& Tanga, P. 2009, P\&SS, 57, 259

DeMeo, F. E., Binzel, R. P., Slivan, S. M., \& Bus, S. J. 2009, Icar, 202, 160

Durech, J., Sidorin, V., \& Kaasalainen, M. 2010, A\&A, 513, A46

Gioia, I. M., \& Luppino, G. A. 1994, ApJS, 94, 583

Lebofsky, L. A., Sykes, M. V., Tedesco, E. F., et al. 1986, Icar, 68, 239

Liu, C.-Y., Doressoundiram, A., Roques, F., et al. 2015, MNRAS, 446, 932

Markwardt, C. B. 2009, in ASP Conf. Ser. 411, Proc. Astronomical Data Analysis Software and Systems XVIII, Nonlinear Least Squares Fitting in IDL with MPFIT, ed. D. Bohlender, P. Dowler, \& D. Durand (San Francisco, CA: ASP), 251

Michalowski, T., Kwiatkowski, T., Kaasalainen, M., et al. 2004, A\&A, 416, 353

Moré, J. 1978, in Numerical Analysis, Vol. 630, ed. G. A. Watson (Berlin: Springer), 105

Muinonen, K. 1989, ApOpt, 28, 3044

Petrov, L., Kovalev, Y. Y., Fomalont, E., \& Gordon, D. 2008, AJ, 136, 580

Rector, T. A., Stocke, J. T., \& Perlman, E. S. 1999, ApJ, 516, 145

Roques, F., Moncuquet, M., \& Sicardy, B. 1987, AJ, 93, 1549

Schlichting, H. E., Ofek, E. O., Sari, R., et al. 2012, ApJ, 761, 150

Tedesco, E. F., Noah, P. V., Noah, M., \& Price, S. D. 2004, IRAS Minor Planet Survey, IRAS-A-FPA-3-RDR-IMPS-V6.0. NASA Planetary Data System, http://sbn.psi.edu/pds/resource/imps.html

Trahan, R., \& Hyland, D. 2014, ApOpt, 53, 3540

Usui, F., Kuroda, D., Müller, T., et al. 2011, PASJ, 63, 1117 